2012 ein ACS auf. Für die interne Qualitätskontrolle existiert ein CPU-Register, finanziell unterstützt von der DGK. Münzel würde sich wünschen, dass die Teilnahme wie bei den "stroke units" ein Muss ist, was bislang nicht so ist. In den letzten Jahren kommen laut inm immer mehr Patienten mit Rhythmusstörungen, hauptsächlich Vorhofflimmern, in die CPU, sodass diskutiert wurde, ob nicht auch Rhythmus-Stationen zu überlegen wären. Die CPUs sollten gut mit den niedergelassenen Ärzten vernetzt sein. Erweisen sich etwa in Mainz die Brustschmerzen als nicht kardial bedingt, werden die Patienten an einen/ihren niedergelassenen Arzt verwiesen.

\section{Szenario Septumruptur}

Der akute Myokardinfarkt ist nicht gerade arm an Überraschungen. Zum Verzweifeln bringt er die Kollegen aber, wenn ein großer Vorderwandinfarkt das Septum in Mitleidenschaft zieht und ein Loch die Folge ist: Infarkt-bedingte Ventrikelseptumdefekte stürzen die Hämodynamik ins Chaos. Ein Shunt zwischen linker und rechter Kammer entsteht, ultimatives Pumpversagen droht. Bisher galt, dass über $80 \%$ dieser Patienten nicht zu retten sind, wenn nicht rasch der Herzchirurg den Septumdefekt verschließt. Liegt der Patient im Schock, kann man von einer 100\%igen Letalität ausgehen, betonte
Prof. Gerhard Schuler, Leipzig. Eine Horrorszenario, zu dem es meist zwei bis sechs Tage nach dem akuten Ereignis kommt. Das ist zwar selten, aber es kommt in 1-2\% der Fälle vor, sodass Herzzentren mit hohem Durchsatz durchaus Zeuge solcher Tragödien werden. Mit den neuen interventionellen Techniken können deutlich bessere Ergebnisse erzielt werden, aber nur, wenn erfahrene Kathetervirtuosen gerade Dienst haben. Dann, so Schuler, ist beim Shuntverschluss eine technische Erfolgsrate von über $80 \%$ zu erzielen und eine Senkung der Letalität auf $50 \%$, sofern noch kein kardiogener Schock vorliegt.

Dr. med. Jochen Aumiller I]

\title{
Man wird ja wohl noch träumen dürfen
}

Der Blick in die Glaskugel ist so eine Sache, gestand Prof. Hans-Reiner Figulla, Jena, als er dazu aufgefordert wurde. Bereits vor gut zehn Jahren hat er sich dazu überreden lassen und hätte mit seinen Tipps in der Tombola höchstens einen Trostpreis bekommen. Damals setzte er voll auf die molekulare Kardiologie mit revolutionären Fortschritten in Diagnostik, Gentherapie und Stammzellen. Der Ingenieurskunst vertraute er fast grenzenlos. Neue Mikromethoden sah er ante portas, aber auch den Durchbruch beim Herzersatz.

Doch man wird ja noch träumen dürfen und so völlig daneben waren die Prophezeiungen auch wieder nicht. Figullas Phantasie hat sich nur großzügig über die Zeitachse hinweggesetzt. Dass er sich vorstellen konnte, dass alles schneller geht, mag an seinen Illusionen gelegen haben, die er selbst noch vor einer Dekade bei der Erfindung und Realisierung so mancher kardiotechnischen Innovationen hatte. Was vielleicht nicht allgemein bekannt ist: Figulla firmiert zwar als Kardiologie-Ordinarius in Jena, er ist international aber vor allem als Erfinder von künstlichen Herzklappen be- kannt. Er hat Patente u.a. für interventionell implantierbare Aortenklappen. Insgesamt dürfte er deutlich über 50 Patente sein eigen nennen. Der Mann hat also Visionen und ist kein Tagträumer. Dennoch ist unser molekulares Krankheitsverständnis weit hinter den Erwartungen zurückgeblieben.

Tatsache heute ist, dass die KHK-Mortalität in den letzten Jahrzehnten eindrucksvoll gesenkt werden konnte. Die Gründe dafür sind zu $40 \%$ auf den therapeutischen Fortschritt und in 54\% auf die Beeinflussung der Risikofaktoren zurückzuführen. Ein Anteil von 6\% sei nicht zu erklären (Earl S. Ford et al. NEJM 2007). Wie lassen sich nun die Erfolgsquoten bei der KHK weiter steigern? Langfristig sicher nicht durch Revaskularisationsprozeduren bei der stabilen KHK, meint Figulla. Prognostische Revaskularisationen hält er nicht für sinnvoll, er sagt sogar einen Rückgang der elektiven $\mathrm{PCl}$ voraus. Es gilt bei der Primärprävention anzusetzen, denn die KHK ist eine Systemerkrankung, keine isolierte Koronaranomalie. „Wir können auch nicht voraussagen, welche Gefäße zugehen werden." So sind präventive antiatherogen Konzepte so- wie effektivere Verfahren zum Risikoscreening (mit Biomarkern oder Genen) wichtig und richtig.

Für die nahe Zukunft erwartet Figulla neue Wege zur LDL-Senkung, etwa humane Antikörper gegen PCSK9 (Subtilisin/Kexin Type 9a). Viele neue Substanzen werden erprobt, LDL-Reduktionen von $70 \%$ scheinen machbar. Wichtig wären auch bessere Ansätze zum Abnehmen, um die Adipositas-Welle aufhalten zu können. Die medikamentösen Ansätze sind noch sehr kritisch zu sehen.

Auf der Wunschliste stehen auch Mittel zur Plaquesstabilisierung (Chemokin-Antagonisten, Rezeptorblocker). Hoffnung macht das Plaque-Imaging, evtl. mit Biomarkern. Viel verspricht sich Figulla von dem nachhaltigen Schutz vor Reperfusionsschäden nach $\mathrm{PCl}$ oder Lyse. Es geht darum, den Radikalenansturm auf die Zellwände bei der Reperfusion abzufangen oder zu neutralisieren. Schließlich zeigt die Glaskugel Fortschritte im biologischen Reparaturbetrieb, falls postischämisch Narben entstehen: Zelltherapie und mechanische Stabilisierung sollen helfen. Die Konsequenzen all der erhofften Fortschritte liegen auf der Hand. Den Patienten geht es besser, nicht aber den Kardiologen. Weniger werden dann reichen, gibt Figulla zu bedenken.

(J.A) II 